\title{
Evaluación de la efectividad biológica de hormonales para incrementar el amarre de frutos en mandarina Fremont (Citrus reticulata Blanco).
}

Evaluation of the biological effectiveness of hormones to increase fruit mooring in Fremont mandarin (Citrus reticulata Blanco).

Vázquez Olmedo David ${ }^{1}$; Rodriguez Cabrera Rocío ${ }^{1}$, Elorza Martínez Pablo ${ }^{1 凶}$; Ramírez Hernández, Teresita ${ }^{2}$; Dávila Lezama María del Rosario ${ }^{2}$.

${ }^{1}$ Facultad de Ciencias Biológicas y agropecuarias campus Tuxpan. Universidad Veracruzana. ${ }^{2}$ Facultad de Ciencias Biológicas y agropecuarias campus Córdoba. Universidad Veracruzana.

${ }^{\bowtie}$ Autor para correspondencia: pelorza@uv.mx

Recibido: 18/02/2017

Aceptado: 30/06/2017

\section{RESUMEN}

El uso de reguladores de crecimiento es una alternativa para incrementar el porcentaje de amarre de frutos, lo cual se traduce en incrementos de la producción. Se evaluaron los efectos de la aplicación de 5 tratamientos entre ellos, 3 diferentes hormonas, un complejo hormonal y un testigo, las hormonas aplicadas correspondieron principalmente a hormonas promotoras del crecimiento. Las variables de respuesta evaluadas fueron: Porcentaje de frutos amarrados por brote, diámetro polar y ecuatorial de los frutos, calidad externa de los frutos color y textura, evaluación de la fitotoxicidad en los árboles y estimación de la producción de fruta. Los resultados de los tratamientos a base de giberelinas y de auxinas, registran mayor promedio y rendimiento de frutos en relación al testigo. La mejor producción de fruta se obtuvo en los tratamientos de auxinas y giberelinas, los cuales superaron al testigo en un $24.33 \%$ y $22.89 \%$, respectivamente. La aplicación de las hormonas evaluadas no afectó, ni modificó la calidad de los frutos: la textura, y el color. No se presentó efecto de fitotoxicidad en ninguno de los tratamientos evaluados.

Palabras clave: Cítricos, Hormonas, Amarre de flor

\begin{abstract}
The use of growth regulators is an alternative to increase the percentage of berthing of fruits, which translates into increases in production. We evaluated the effects of the application of 5 treatments among them, 3 different hormones, a hormonal complex and a control, the hormones applied corresponded mainly to growth promoting hormones. The variables evaluated were: Percentage of fruits moored by shoot, polar and equatorial diameter of fruits, external quality of fruits color and texture, evaluation of phytotoxicity in trees and estimation of fruit production. The results of treatments based on gibberellins and auxins, recorded higher average and fruit yield in relation to the control. The best fruit production was obtained in auxin and gibberellin treatments, which surpassed the control by $24.33 \%$ and $22.89 \%$, respectively. The application of the hormones evaluated did not affect or modify the quality of the fruits: texture, and color. There was no phytotoxicity effect in any of the evaluated treatments.
\end{abstract}

Keywords: Citrus, Hormones, Flower Moor 


\section{INTRODUCCIÓN}

La producción de los agrios depende, críticamente de la floración, lo que es norma general, en la mayor parte de las plantas cultivadas. El cuajado es el factor central en la determinación de la cosecha en los agrios y este se halla directamente relacionada con el número de frutos cosechados, y el producto de este por el peso individual de los frutos, determina su cuantía final. Como en otros frutales, el amarre o cuajado y el desarrollo de los frutos es consecuencia de factores endógenos y exógenos. Entre los primeros, son las características genéticas de la especie y de la variedad, junto con los factores fisiológicos, los que determinan la producción y su calidad; entre los segundos, las condiciones del medio y el cultivo. Los factores genéticos y las condiciones del medio, suelo y clima, no pueden ser alterados en condiciones de cultivo. Los factores fisiológicos, por el contrario, pueden ser modificados. Las técnicas de cultivo tienen este objetivo como fin: aplicar los conocimientos sobre el desarrollo de las plantas para lograr una mejor producción y calidad de sus frutos (Agustí et al., 2003). Desde un punto de vista fisiológico, los factores nutrimentales y hormonales son determinantes en la producción y calidad de los frutos. Los primeros hacen referencia, prioritariamente, a la disponibilidad por carbohidratos; los segundos determinan la capacidad sumidero del fruto $\mathrm{y}$, por tanto, su poder para atraerlos. Cuando uno de ellos, o los dos, son deficitarios, la producción y el desarrollo del fruto se reducen, dependiendo del estado de desarrollo del fruto en que se produzca el déficit (Agustí et al., 2003).

El objetivo de la investigación fue: Evaluar el efecto del uso de diferentes fitohormonas en el amarre de frutos de mandarina Fremont.

El trabajo de investigación fu realizado en la región norte del estado de Veracruz, en la comunidad Morgadal, del municipio de Papantla de Olarte Veracruz. El área total de estudio donde fue de $2,160 \mathrm{~m}^{2}$, teniéndose como unidad experimental una superficie de $108 \mathrm{~m} 2$ y como parcela útil 36 $\mathrm{m} 2$. El diseño experimental utilizado en el estudio fue el modelo de bloques completos al azar, evaluando 5 tratamientos con 4 repeticiones, por cada uno de los tratamientos. Cada unidad experimental fue representada por tres árboles de los cuales solo el árbol de en medio será el utilizado para la toma de datos del experimento.

Los tratamientos con los mejores promedios de amarre de frutos fueron el de auxinas y el giberelinas. Para la variable diámetro ecuatorial hubo diferencias en el tratamiento tres que es el complejo hormonal a los 45 días con $17.10 \mathrm{~mm}$; a los 60 días con 23.015; a los 75 días con $27.812 \mathrm{~mm}$ y a los 90 DDPA con $31.171 \mathrm{~mm}$. Asimismo el tratamiento dos que son las auxinas a los 75 días con $27.593 \mathrm{~mm}$ y a los 90 DDPA con $30.859 \mathrm{~mm}$, en relación con el testigo que se tuvo un resultado de $16.953 \mathrm{~mm}$ a los 45 días; de $22.671 \mathrm{~mm}$ a los 60 días; de $27.390 \mathrm{~mm}$ a los 75 días y de $30.750 \mathrm{~mm}$ a los 90 DDPA.

en los resultados de esta investigación a los 90 DDPA, el numero de frutos que va de los 732.5 hasta los 947.5 (cuadro 8), no esta relacionado con el diámetro polar de 28.67 hasta $29.45 \mathrm{~mm}$ (figura

3) y el diámetro ecuatorial de 29.78 a $31.75 \mathrm{~mm}$ (figura 4), ya que se presentan diámetros afines independientemente del numero de frutos., lo cual difieren con lo mencionado por Agusti (2004) donde afirma que el tamaño del fruto esta inversamente relacionada con el numero de frutos por árbol. Los resultados antes mencionados de este trabajo no concuerdan con lo mencionado por (Agusti, 2003) donde afirma que la aplicación de auxinas se ha relacionado con el proceso de elongación celular (fase II del desarrollo del fruto) mas que con el proceso de división celular o cuajado. En relación a las características físicas de los frutos de acuerdo a los datos obtenidos de las evaluaciones realizadas, se obtuvo como resultado frutos de textura rugosa, lo cual difiere con lo mencionado por Cruz (2013) donde menciona que la textura final del fruto es de apariencia semi-rugosa, cabe señalar que la información que se presenta de los frutos en este periodo, en el término de la última evaluación no presentaban el tamaño final de su crecimiento por lo que la apariencia y el grosor de su cascara era más rustica, a medida que el crecimiento del fruto siga su ritmo, se seguirán produciendo cambios en su textura.

Con base a la escala de evaluación empleada para el parámetro de coloración de la fruta, no se apreciaron diferencias entre los tratamientos en estudio. Calificándose un color

Revista Científica Biológico Agropecuaria Tuxpan 5 (1) ISSN: 2007-6940 
verde en las evaluaciones a los 30 y 45 DDPA. En las evaluaciones posteriores entre los 60, 75 y 90 DDPA, los frutos de todos los tratamientos tuvieron una tonalidad verde intenso.

Los resultados logrados, reflejan que no hubo influencia sobre la coloración de los frutos en ninguno de los tratamientos en comparación. En la variable fitotoxicidad no presentó grado de fitotoxicidad en ningún tratamiento ni en la fecha de toma de datos, reportando un valor de 0, Esto concuerda con los resultados presentados por Rodríguez et al. (2007) quienes registraron $0 \%$ de fitotoxicidad en el tratamiento aplicado, en brotes, hojas, y frutos, aun cuando sus aplicaciones en auxinas fueron durante dos años consecutivos. Sin embargo, difiere con lo reportado por Retamal (2003), donde observó una leve deformación en algunas hojas en los brotes nuevos, que consistió en una epinástia y amarillamiento de los bordes en la aplicación de auxinas.

Para la variable, estimación de rendimiento de fruta, el análisis realizado de la comparación de medias por el método de Tukey, en los tratamientos evaluados, indica que no se encontraron diferencias estadísticas entre ellos, por lo tanto, la producción de fruta por hectárea fue semejante desde el punto estadístico. Sin embargo, al efectuar la comparación con base a los promedios de producción, se aprecia a dos de los tratamientos como los mejores en cuanto a producción, siendo éstos, el tratamiento 2 con aplicación de auxinas con una producción de fruta de 36,198.36 Ton/ha, y el tratamiento 4 a base de giberelinas el cual tuvo una producción de fruta estimada de 35,521.30 Ton/ha, los cuales superaron al tratamiento testigo por $8,806.53$ y $8,129.47$, Ton /ha, respectivamente.

\section{CONCLUSIONES}

Los efectos del uso de diferentes fitohormonas presentan un efecto positivo incrementando más amarre de frutos en mandarina Fremont. Considerando el promedio de los tratamientos, se aprecia una tendencia favorable en el amarre de frutos, desde la primera hasta la última evaluación en los tratamientos a base de giberelinas y auxinas, los cuales superan en un 3 por ciento en relación al testigo.

El porcentaje de amarre obtenido en los tratamientos a base de hormonas se considera bajo con relación a otros estudios, esto quizás se debió a las condiciones de sequía que prevalecieron en la región en el periodo en que se desarrolló el estudio. El tamaño de los frutos no se vio influenciado por ninguno de los tratamientos en comparación, durante los días observados.

La coloración de los frutos no fue modificado por alguno de los tratamientos en estudio. Ninguno de los tratamientos ocasionó algún efecto de fitoxicidad en brotes vegetativos, flores ni en los frutos.

\section{BIBLIOGRAFIA}

Agustí, M. y Almela, V. 1991. Aplicación de fitoreguladores en citricultura. Editorial Aedo. Barcelona, España. 261p.

Agustí, M. 2004. Fruticultura. Editorial: Mundi Prensa. 493 p.

Agustí, M. 2003.Citricultura.Editorial: Mundiprensa.España.422 p.

Morín, L. Charles.1983. Cultivo de cítricos.- $2^{\mathrm{a}}$. Ed.; reimpresión - san José Costa Rica: IICACIDIA, 1983. 607 p.

Agustí M, Martínez-Fuentes, Mesejo C, Juan Vicente M., Almela V. 2003.Cuajado y desarrollo de los frutos cítricos. Valencia. Edita: Generalitat Valenciana .Consellería de Agricultura, Pesca y Alimentación.

Cruz, S.S.2011. Manual de mandarinas cultivadas en la región nororiental del estado de puebla. Fundación produce puebla a.c.42.p.

Díaz, M.D. 2002. Fisiología de los árboles frutales .AGT EDITOR, S.A.385 p.

Squeo,F,A.\& Cardemil,L. 2007.Fisiologia vegetal. Ediciones: Universidad de la Serena. La serena chile.p.28.

Guardiola, J.L.1998. Fructificación, producción y tamaño del fruto. Sociedad Gardiazábal y Magdahl Ltda. Seminario internacional de cítricos. Viña del Mar, 13-15 de mayo. pp. 159- 194.

Retamal, O. 2003. Efecto de la aplicación de auxinas de síntesis sobre la producción y calibre 
final en el fruto del naranjo (Citrus sinensis (L.)Osbeck) cv. Tardía de valencia. Taller de licenciatura, Ing. Agr. Quillota, Universidad Católica de Valparaíso, Facultad de Agronomía. 58 $\mathrm{P}$.

Osuna, E. T; Contreras, A. L. A; Contreras, M. R. 2011. Uso de Fitoreguladores en la citricultura. Tercera Jornada de Transferencia de Tecnología de Cítricos. Fundación Produce de Sinaloa A.C.SAGARPA-Gobierno de Sinaloa. México pp. 1926.

Rebolledo, R.A .2012. Fisiología de la floración y fructificación en los cítricos. Corporación Universitaria Lasallista.18 p.

Rodriguez,A.V;M,S.M;M.G.C.2007. Uso de reguladores de crecimiento para incrementar la productividad de mandarino 'Clemenules'. Rev. Bras. Frutic., Jaboticabal. volumen29.48-56 p. https://doi.org/10.1590/S0100-29452007000100012

Vidal, C. A. E. 2006. Efecto de la aplicación de GA3 y 3, 5, 6 TPA, sobre la productividad y calidad externa en el mandarino Clementino (Citrus clementina Blanco) cv. Clemenules (Tesis Ing. Agr). Universidad Católica de Valparaíso. Fac. de Agronomía. Chile.

SIAP.2014.Servicio de información agroalimentaria y pesquera. Consulta: cierre de la producción agrícola por estado.consultado:18-122015.

SIAP. 2014 Servicio de información agroalimentaria y pesquera .consulta superficie total de cítricos en Veracruz. Consultado: 18 -12 -2015. 
Copyright (c) 2017 David Vázquez Olmedo, Rocio Rodriguez Cabrera, Pablo Elorza Martinez,

Teresita Ramirez Hernández y Maria del Rosario Dávila Lezama

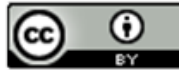

Este tex to está protegido por una licencia licencia Creative Commons 4.0

Usted es libre para Compartir —copiar y redistribuir el material en cualquier medio o form ato- y Adaptar el documento —remezclar, transformar y crear a partir del material- para cualquier propósito, incluso para fines comerciales, siempre que cumpla la condición de:

Atribución: Usted debe dar crédito a la obra original de manera adecuada, proporcionar un enlace a la licencia, e indicar si se han realizado cambios. Puede hacerlo en cualquier form a razonable, pero no de form a tal que sugiera que tiene el apoyo del licenciante o 10 recibe por el uso que hace de la obra.

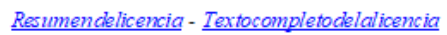

